\title{
Técnicas de conservação pós-colheita para o manjericão
}

TEIXEIRA, D.A. ${ }^{*}$; GOMES, J.A.O..'; BONFIM, F.P.G. ${ }^{1}$; PARDO, P.I. ${ }^{2}$ MAYOBRE, M.T. ${ }^{3}$

${ }^{1}$ UNESP, Faculdade de Ciências Agronômicas, Departamento de Horticultura, Fazenda Lageado, Portaria I: Rua José Barbosa de Barros, $n^{\circ}$ 1780, Cx. Postal 237, CEP 18.610-307- Botucatu, SP; 2Universidad se Santiago de Compostela, Escola Técnica Superior de Enxeñaría, Rúa Lopez Gómez de Marzoa s/n, Campus Vida, CEP 15.782- Santiago de Compostela, Espanha, ${ }^{3}$ Universidad de La Republica, Falcultad de Agronomia, Avenida Garzón, $n^{\circ}$ 780, CEP 12.800 - Montevidéo, Uruguai. *Autor para correspondência: daniela.teixeira@hotmail.com

\begin{abstract}
RESUMO: O trabalho teve como objetivo avaliar a qualidade e vida útil de ramos de manjericão submetidos a procedimentos de conservação pós-colheita. $O$ experimento foi conduzido no Laboratório de Plantas Medicinais do Departamento de Horticultura, da Universidade Estadual Júlio de Mesquita Filho, município de Botucatu, São Paulo. O delineamento utilizado foi inteiramente casualizado com quatro tratamentos e seis repetições. Os tratamentos consistiram de técnicas de conservação pós-colheita seguintes: Testemunha (sem tratamento pós-colheita), embalagem plástica, hidroresfriamento, hidroresfriamento + embalagem. As variáveis analisadas foram perda de massa e vida útil. Os dados foram submetidos à análise de variância e as médias comparadas pelo teste de Tukey a $5 \%$ de probabilidade, pelo programa ASSISTAT 7.7 beta. A partir desses dados, a utilização de embalagem plástica associada ao método de hidroresfriamento contribuiu para a conservação pós-colheita e vida útil do manjericão.
\end{abstract}

Palavras-chave: vida útil, armazenamento, embalagem.

\begin{abstract}
Postharvest conservation techniques for basil. The study aimed to evaluate which post-harvest technique provided better quality and life of basil branches The experiment was conducted on Medicinal Plants Laboratory of the Department of Horticulture, da Universidade Estadual Júlio de Mesquita Filho, Botucatu, São Paulo.The design was completely randomized with four treatments and six repetitions. The treatments consisted of post-harvest preservation techniques: Control (without post-harvest treatment), plastic packaging, hydrocooling, hydrocooling + package. The variables analyzed were mass loss and lifespan. Data were subjected to analysis of variance and means compared by Tukey test at $5 \%$ probability, the ASSISTAT 7.7 beta program. From these data, it was concluded that the use of plastic packaging associated with hydrocooling contributed method for post-harvest storage lifespan and basil.
\end{abstract}

Keywords: lifespan, storage, packaging

\section{INTRODUÇÃO}

O manjericão (Ocimum basilicum L.) é comercialmente cultivado para utilização de suas folhas verdes e aromáticas, as quais são usadas frescas ou secas como aromatizante ou tempero (Loughrin \& Kasperbauer, 2001). É empregado tanto na indústria culinária, quanto na fitoterápica e na medicina popular devido ao teor e composição do seu óleo essencial (Silva et al., 2005) . O óleo essencial de manjericão com alta concentração de linalol é valorizado no mercado internacional e amplamente usado nas indústrias de condimentos e cosméticos (Carvalho Filho et al., 2006). Apresenta propriedades inseticidas, repelentes, antimicrobianas sendo também utilizado na conservação de grãos (Fernandes, 2004). Os produtos hortícolas, em especial as hortaliças e plantas medicinais aromáticas folhosas, iniciam acelerado processo de deterioração logo após a colheita. A vida útil é determinada pela combinação de fatores internos e externos aos órgãos vegetais (Finger \& Vieira, 1997).

Algumas técnicas simples de pós-colheita podem prolongar a vida de prateleira das hortícolas sem resultar em grandes custos ao produtor, como a utilização de embalagens plásticas, as quais funcionam como proteção física reduzindo a 
deterioração pós-colheita devido à perda de água (Finger \& Vieira, 1997) e o pré-resfriamento, o qual é particularmente benéfico aos produtos altamente perecíveis, sendo essencial na remoção do calor de campo dos produtos recém-colhidos (Wills et al., 2004).

Reduzir a temperatura do vegetal pode ser capaz de maximizar a vida útil na póscolheita de flores, pois retarda os processos metabólicos responsáveis pela senescência. $O$ rápido resfriamento destes produtos, logo após a colheita, reduz o calor de campo e diminui a atividade metabólica (Gillies \& Toivonen, 1995). $O$ pré-resfriamento tem se mostrado eficaz na conservação de produtos hortícolas, tendo destaque o hidroresfriamento. A técnica consiste na imersão do vegetal em água gelada para diminuir a temperatura antes que seja embalado. Este método evita também a perda da umidade durante os processos posteriores de beneficiamento (Wills et al., 2004).

A embalagem adequada confere ao produto uma proteção no manuseio e transporte, além de auxiliar no aumento da vida útil, pois mantem a umidade relativa no seu interior, o que confere melhores características para a comercialização. Filmes plásticos podem estender a vida útil de produtos sensíveis e altamente suscetíveis ao manuseio (Shibairo et al., 1997). Sendo assim, o objetivo do trabalho foi verificar a técnica que proporciona melhor qualidade aos ramos de manjericão.

\section{MATERIAL E MÉTODOS}

O experimento foi conduzido no Laboratório de Plantas Medicinais do Departamento de Horticultura, da Universidade Estadual Júlio de Mesquita Filho, no município de Botucatu, no Estado de São Paulo, na primeira quinzena de novembro de 2013. O delineamento utilizado foi inteiramente casualizado com quatro tratamentos e seis repetições. Os tratamentos consistiram das técnicas de conservação pós-colheita seguintes: Testemunha (sem tratamento pós-colheita), embalagem plástica, hidroresfriamento, hidroresfriamento + embalagem. Ramos frescos de manjericão, provenientes do horto medicinal da Faculdade de Ciências Agronômicas - UNESP/ Botucatu, foram colhidos, selecionados, pesados e separados em 25 gramas, seguindo padrões de comercialização. O material vegetal foi depositado no Herbário Irina Delanova Gemtchujnicov, Universidade Estadual Paulista, Botucatu-SP, sob registro BOTU 26037.

Os tratamentos com embalagem foram colocados em sacos plásticos perfurados para minimizar os efeitos da transpiração. Para o hidroresfriamento os ramos foram imersos em água a $5^{\circ} \mathrm{C}$ por 16 minutos. Foi realizado um préteste para determinar o tempo de pré-resfriamento, no qual as folhas foram submersas à mistura de gelo e água à $5^{\circ} \mathrm{C}$, a temperatura foi medida com termômetro digital infravermelho (ST-600 Incoterm). As condições de armazenamento dos ramos foram: temperatura ambiente de $21,84{ }^{\circ} \mathrm{C}$ e umidade relativa do ar 69,05\%, segundo dados da Estação Meteorológica do Departamento de Solos e Recursos Ambientais da Universidade Estadual Júlio de Mesquita Filho, no município de Botucatu. As variáveis analisadas foram perda de massa $(\mathrm{g})$ avaliada diariamente e vida útil. Quando as partes vegetais apresentaram sinais de deterioração, como mudanças de coloração, murcha, indicando estar imprópria à comercialização, a avaliação da vida útil foi suspensa.

As avaliações das características visuais ocorreram a cada 24 horas de armazenamento. Os ramos foram avaliados até o tempo final (fim da vida útil).

\section{RESULTADOS E DISCUSSÃO}

$\mathrm{Na}$ determinação do tempo de préresfriamento por imersão em água à $5^{\circ} \mathrm{C}$ observou-se inicialmente queda brusca na temperatura das folhas com o aumento do tempo. A partir de 16 minutos de hidroresfriamento, a temperatura permaneceu constante devido ao equilíbrio das temperaturas conforme descrito por Wills et al. (2004).

Os ramos hidroresfriados + embalados apresentaram o maior tempo de vida útil (168 horas), enquanto que aqueles que foram apenas embalados perderam suas características após 120 horas de armazenamento (Tabela 1).

Para perda de massa, durante todo o período de armazenamento, as menores médias ocorreram nos ramos de manjericão hidroresfriados+embalados, mostrando a importância da embalagem na redução de perda de água e consequente manutenção da turgescência dos ramos quando associada a técnica de diminuição de temperatura. A perda de massa observada nos ramos hidroresfriados quando comparados aos ramos controle foram semelhantes entre si. Esse efeito esta relacionado

TABELA 1. Vida útil de ramos de manjericão em função dos tratamentos

\begin{tabular}{ll}
\hline Tratamentos & Vida Útil \\
\hline Testemunha & 24 horas \\
Hidroresfriamento & 48 horas \\
Embalagem & 120 horas \\
Hidroresfriamento+Embalagem & 168 horas \\
\hline
\end{tabular}


TABELA 2. Valores médios de perda de massa dos ramos $(\mathrm{g})$ de manjericão em função do tempo de armazenamento

\begin{tabular}{ccccc}
\hline Tempo(h) & Testemunha & Hidroresfriamento & Embalagem & Hidroresf.+Embalagem \\
\hline 24 & $28.05 \mathrm{~d}$ & $36.45 \mathrm{c}$ & $49.23 \mathrm{~b}$ & $62.15 \mathrm{a}$ \\
48 & $28.08 \mathrm{c}$ & $22.21 \mathrm{c}$ & $46.40 \mathrm{~b}$ & $59.21 \mathrm{a}$ \\
72 & $14.78 \mathrm{c}$ & $16.23 \mathrm{c}$ & $44.53 \mathrm{~b}$ & $57.70 \mathrm{a}$ \\
96 & $14.78 \mathrm{c}$ & $16.23 \mathrm{c}$ & $44.53 \mathrm{~b}$ & $57.70 \mathrm{a}$ \\
120 & $9.66 \mathrm{c}$ & $10.31 \mathrm{c}$ & $40.60 \mathrm{~b}$ & $54.30 \mathrm{a}$ \\
144 & $9.03 \mathrm{c}$ & $9.58 \mathrm{c}$ & $38.72 \mathrm{~b}$ & $52.76 \mathrm{a}$ \\
168 & $8.5 \mathrm{c}$ & $8.85 \mathrm{c}$ & $36.85 \mathrm{~b}$ & $51.23 \mathrm{a}$ \\
\hline
\end{tabular}

à perda por evaporação da água retida nas folhas ou absorvida durante o hidroresfriamento (Tabela 2), como observado por Gillies \& Toivonen (1995) estudando brócolis.

O hidroresfriamento por utilização de água gelada possui alta eficiência, devido ao alto calor específico da água e sua taxa de transferência de calor (Delgado \& Sun, 2001). Segundo Teruel et al. (2002),o hidroresfriamento pode promover o abaixamento da temperatura três vezes mais rápido em relação a outros procedimentos. Outra vantagem desse método é a ausência de desidratação, além de poder ocorrer à absorção de água pelo produto (Henry \& Bennett, 1973).

Devido a curta vida útil que algumas hortaliças e plantas medicinais apresentam, estas necessitam ser resfriadas rapidamente, como o brocólis (Brosnan \& Sun, 2001). Segundo Golob et al. (2002), como consequência a rápida redução do metabolismo, o produto reduz a perda de matéria seca e também a perda de água, promovendo acréscimo de longevidade. No presente estudo foi utilizado o resfriamento seguido à manutenção do material em condições ambientes, diferentemente do experimento de Álvarez (2007) onde se observou que o pré-resfriamento com imersão em gelo e água a $5^{\circ} \mathrm{C}$ por 15 minutos o material permaneceu sob refrigeração, estendeu a vida pós-colheita de salsinha (Petroselinum crispum). O pré-resfriamento de salsa reduziu a perda de massa e retardou o início da perda de turgidez das folhas, no entanto, este efeito benéfico foi acentuadamente reduzido ao armazenarem-se os maços a $25{ }^{\circ} \mathrm{C}$ (Álvares \& Negreiros, 2010). Alfaces (Lactuca sativa) armazenadas sob refrigeração e acondicionadas em embalagens plásticas de polietileno de baixa densidade, conservaram a qualidade aparente por mais tempo (Reis et al., 2014).

Chitarra \& Chitarra (2005) citam que o emprego de filmes plásticos, limita as trocas gasosas e a perda de água dos alimentos para o ambiente, reduzindo o metabolismo do produto e prolongando sua vida pós-colheita. As maiores alterações fisiológicas, bioquímicas e moleculares de vários produtos como o brócolis (Galvão, 2005) ocorrem nas primeiras horas após a colheita. O efeito do pré-resfriamento sobre a redução da perda de massa de brócolis nas primeiras 24 horas foi explicado pela redução do gradiente de pressão de vapor e da taxa de difusão do vapor de água do produto para o ambiente já que, como citado por Taiz \& Zeiger (2004), a perda de umidade de produtos frescos é grandemente determinada pela diferença entre a pressão de vapor do produto e a pressão de vapor do ar circundante. Portanto, como a manutenção da qualidade comercial é de vital importância, é necessário não somente resfriar o produto, mas também embalá-lo o mais rápido possível após a colheita. O tempo de resfriamento varia proporcionalmente com o volume do produto, sendo as hortaliças resfriadas de 1,5 até 55 minutos (Teruel et al., 2004). Nota-se, desta forma, a grande importância do uso da embalagem plástica na redução da perda de massa do produto. Messias (2004) observou efeito do mesmo tipo de embalagem na redução de $6 \%$ da perda de massa acumulada de folhas frescas de manjericão cv. semi-roxo e cv. branco, armazenadas a $5^{\circ} \mathrm{C}$. Em condições ambientes a temperatura mais alta ocasiona um aumento do gradiente de pressão de vapor, a atividade metabólica e a troca gasosa com o meio, elevando consequentemente a respiração e reduzindo a conservação dos produtos hortícolas (Taiz \& Zeiger, 2004).

\section{CONCLUSÕES}

A utilização de embalagem plástica associada ao método de hidroresfriamento contribuiu, significativamente, para a conservação pós-colheita do manjericão, obtendo assim, vida útil de até sete dias.

\section{REFERÊNCIAS}

ÁLVAREZ, V.S. et al. Effect of pre- cooling postharvest of parsley leaves. Journal of Food Agriculture and Environmental, v. 5, n. 2, p. 31-34, 2007.

Rev. Bras. PI. Med., Campinas, v.18, n.1, p.168-171, 2016. 
ÁLVARES, V.L.; NEGREIROS, J.R.S. Pre-cooling and packing in the conservation of parsley leaves. Brazilian Journal Food Technology, v. 13, n. 2, p. 107-111, 2010.

BROSNAN, T.; SUN, D.W. Precooling techiniques and applications for horticultural products- a review. International Journal Refrigeration, v.24, p.154-170, 2001.

CARVALHO FILHO, J.L.S. et al. Influence of the harvesting time, temperature and drying period on basil (Ocimum basilicum L.) essential oil. Revista Brasileira de Farmacognosia. v.16, n.1, p. 24-30, 2006.

CHITARRA, M.I.F.; CHITARRA, A.B. Pós-colheita de frutas e hortaliças: fisiologia e manuseio. 2 ed. Editora UFLA, Lavras, Brasil, 2005, 785 p.

DELGADO, A.E.; SUN, D.W. Heat and mass transfers models for predicting freezing process e a review. Journal of Food Engineering. v. 47,n.3, p.157-174, 2001.

FERNANDES, P.C. et al. Cultivo de manjericão em hidroponia e em diferentes substratos sob ambiente protegido. Horticultura Brasileira. v.22,n.2, p.260264,2004.

FINGER, F.L.; VIEIRA, G. Controle da perda póscolheita de água em produtos hortícolas. Editora UFV, Viçosa, Brasil. 1997, 29 p.

GALVÃO, H.L. Efeito do pré-resfriamento e do filme de PVC sobre a qualidade pós-colheita de brócolis. 2005. 38 p. (Dissertação de Mestrado). - Universidade Federal de Viçosa, Viçosa, Brasil.

GILLIES, S.L.; TOIVONEN, P.M.A. Cooling methods influences the postharvest quality of broccoli. HortScience, v.30, n.2, p. 313-315,1995.

GOLOB, P. et al. Postharvest science and technology, principles and practices, v, 1. Blackwell Science, New Jersey, 2002, 554 p.

HENRY, F.E.; BENNET, A.H. Hydrocooling vegetables products unit loads. Transactions of ASAE, v. 16, n.4, p.731-733, 1973.

LOUGHRIN, J.H.; KASPERBAUER, M.J.L. Light reflected from colored mulches affects aroma and phenolic content of sweet basil (Ocimum basilicum L.) leaves. Journal of Agricultural and Food Chemistry, v.49, n.3, p.1331-1335, 2001.

MESSIAS, U. Resposta pós-colheita à injúria por frio de três cultivares de manjericão (Ocimum basilicum L.) 2004. 36p. (Dissertação de Mestrado) - Universidade Federal de Viçosa, Viçosa, Brasil.

REIS, H.F. et al. Conservação pós-colheita de alface crespa, de cultivo orgânico e convencional, sob atmosfera modificada. Horticultura Brasileira, v. 32, n.3, 303-309, 2014.

SILVA, S. et al. Basil conservation affected by cropping season, harvest time and storage period. Pesquisa Agropecuária Brasileira, v. 40, n. 4. p. 323-328, 2005.

SHIBAIRO, S.I. et al. Postharvest moisture loss characteristics of carrot (Daucus carota L.) cultivars during short-term storage. Scientia Horticulturae, v. 71, n. 1, p. 1-12, 1997.

TAIZ, L.; ZEIGER, E. Fisiologia vegetal. 3 ed. Trad. Santarém, E. R. et al. Artmed ,Porto Alegre, Brasil. 2004, 719 p.

TERUEL, B. et al. Transferência de calor durante o resfriamento de frutas com ar forçado e com água. Brazilian Journal of Food Technology, v.5, n.90, p.131-138, 2002.

TERUEL, B. et al. Estudo comparativo do resfriamento de laranja Valência em três sistemas de resfriamento. Revista Brasileira de Engenharia Agrícola e Ambiental, v. 5 n.3, p. 481-486. 2004.

WILLS, R. et al. Postharvest: an introduction to the physiology \& handling of fruit, vegetables \& ornamentals. New South Wales University Press, Wallingford, , 2004. 262 p. 